\section{RG Dresden}

Auskunft:

RAin Susanne Köhler

Kanzlei Staab und Kollegen

Schandauer Str. 43

01277 Dresden

Fon: 0351316780 (d)

E-Mail:koehler@staab-dresden.de

Veranstaltungen:

Stammtisch jeweils am vierten Donnerstag im Monat ab 19.00 Uhr (Beginn der Vorträge 19.30 Uhr) im Restaurant La Villetta, Augsburger Str. 43 in 01309 Dresden, Fon: 0351315990.

\section{Landesverband Sachsen-Anhalt \\ Auskunft: \\ Dr. Afra Waterkamp-Faupel \\ Mörickestr. 20 \\ 39114 Magdeburg \\ Fon: 03917315801 (p) \\ E-Mail:waterkamp-faupel@freenet.de}

\section{RG Halle/Saale}

Auskunft:

RAin Sabine Kuhlenkamp

Kanzlei Jürges, Knop \& Stiller

Am Steintor 19

06112 Halle/Saale

Fon: 03452023038 (d)

Fax: 034512298877 (d)

E-Mail:kuhlenkamp@ra-juerges.de

Veranstaltungen:

Stammtisch an jedem dritten Donnerstag im Monat ab 20.00 Uhr in der Gasthaus- brauerei „Zum Schad“, Reilstr. 10, 06114 Halle, Fon: 03455230366.

\section{RG Magdeburg}

Auskunft:

Ruth Störtenbecker

Fon: 01791412467 (p)

Fon: 03915676700 (d)

E-Mail: ruth.stoertenbecker@stk.Isa-net.de Veranstaltungen:

In aller Regel am letzten Donnerstag im Monat um 19.00 Uhr im Marlene, Breiter Weg 202, 39104 Magdeburg (direkt neben dem neuen Justizzentrum).

\section{Landesverband Schleswig-Holstein}

RG Kiel

Auskunft:

Ingeborg Kerssenfischer

Fon: 04349914141 (p)

E-Mail: rg-kiel@djb.de

Veranstaltungen:

Treffen (meist mit Vortrag) am letzten Mittwoch jedes Monats um 19.30 Uhr im Restaurant „Seeburg“, Düsternbrooker Weg 2 in Kiel (ggf. abweichenden Treffpunkt entnehmen Sie bitte der djb-Internetseite).

\section{RG Lübeck}

Auskunft über die Bundesgeschäftsstelle des djb, Fon: 030 4432700,

E-Mail: geschaeftsstelle@djb.de.

Derzeit unregelmäßige Treffen.

\section{Landesverband Thüringen}

Auskunft:

Christina Lorenz

Konrad-Zuse-Str. 11/606

99099 Erfurt

Fon: 03614224726 (p)

Veranstaltungen:

Regelmäßige Treffen jeden zweiten Dienstag im Monat. Ort bitte bei Frau Lorenz erfragen.

\section{Ausland}

\section{RG Brüssel}

Auskunft:

Margarete Hofmann

67, Avenue Michel-Ange

B-100o Brüssel

Fon: +322 2981710 (d)

E-Mail: Margarete.Hofmann@ec.europa. $\mathrm{eu}$

Veranstaltungen:

Abwechselnd Vortragsveranstaltungen in der Landesvertretung Niedersachsen und Stammtisch (allgemeiner Stammtisch oder Stammtisch Junge Juristinnen) im Restaurant Las Castanuelas bzw. Café Flagey im monatlichen Rhythmus. Einladungen und Informationen werden per E-Mail versandt. Weitere Informationen auch unter www.djb.de - RG Brüssel.

\title{
Veranstaltungshinweise
}

\section{Feministischer Juristinnentag}

\section{8. bis 10. Mai 2009, Wannseeforum Berlin}

Das Programm umfasst in den Foren die aktuellen Reformen im Familienrecht, rechtspolitische Diskussionen um Reproduktionstechnologien und Grundsatzfragen staatlicher Integrationspolitik. Weiterhin gibt es zahlreiche Arbeitsgemeinschaften zu aktuellen rechtspraktischen, rechtspolitischen und rechtstheoretischen Fragen wie z.B. zur Behindertenrechtskonvention, zu Strategien zur Durchsetzung der Entgeltgleichheit, zur Forderung nach einer gesetzlichen Regelung der Frauenhausfinanzierung, zu Entwicklungen im europäischen Antidiskriminierungsrecht oder zum Bezug zwischen feministischer und materialistischer Rechtstheorie.

Weitere Informationen:

http://www.feministischer-juristinnentag.de/ 


\section{8. djb-Kongress mit Mitgliederversammlung}

\section{4.-27. September 2009 in Karlsruhe}

Die Lebensumstände und die notwendige Integration von in Deutschland lebenden Migrantinnen und Migranten hat in den letzten Jahren zunehmend Aufmerksamkeit erlangt. Migration und Integration wurden als zentrale Zukunftsthemen der Politik in Bund, Ländern und Gemeinden sowie in der öffentlichen Diskussion erkannt. Auch die Bundesregierung hat sich verstärkt dieser Thematik angenommen (Integrationsgipfel, Integrationsplan, Islamkonferenzen etc.). Jedoch wird auf die spezifischen Probleme, mit denen Migrantinnen konfrontiert werden, überwiegend nur im Zusammenhang mit Gewalt gegen Migrantinnen und Zwangsverheiratungen eingegangen - der djb hat mehrfach Stellung genommen. Das Thema Migration unter der Geschlechterperspektive wird hingegen noch wenig diskutiert.

Unser 38. Bundeskongress mit Eröffnungsveranstaltung am Donnerstag und anschließender Tagung in Vorträgen und Workshops dem Thema der rechtlichen Rahmenbedingungen für eine Integration von Migrantinnen in die deutsche Gesellschaft annehmen. Hierzu gehören insbesondere Fragen der Anerkennung von Bildungsabschlüssen, Vereinbarkeit eines von den verschiedenen Ausformungen der Scharia geprägten Familien- und Erbrecht mit dem deutschen Recht und der Zugang zu Sozialschutz und gesetzlicher Alterssicherung sowie die Frage des Familiennachzugs und des eigenständigen Aufenthaltsrechts nach den Reformen im Zuwanderungsrecht.

Zur Eröffnungsveranstaltung im Bundesverfassungsgericht am 24. September 2009 haben der Präsident des Bundesverfassungsgerichts Prof. Dr. dres. h.c. Jürgen Papier, Bundesjustizministerin Brigitte Zypries und Justizminister Prof. Dr. Ulrich Goll Grußworte zugesagt. Die Richterin des Bundesverfassungsgerichts Prof. Dr. Gertrude LübbeWolff wird die Festrede zum Thema „Integration und Verfassung" halten.

Einladung und Programm werden Sie in der djbZ 2/2009 finden, sowie ab Mai 2009 online auf unserer Webseite www.djb.de.

\section{EWLA-Kongress 2009}

\section{3.-4. Juli 2009 in Reykjavík}

Der diesjährige Kongress der European Women Lawyers Association (EWLA) findet in Island statt. Er steht unter dem Thema „Fundamental Rights and Financial Markets“ - besonders passend für einen Kongress in einem Land, in dem jetzt vorzugsweise Frauen damit betraut werden, den überwiegend von Männern gemachten finanziellen Zusammenbruch zu beheben. Angesichts der in allen europäischen Staaten erwarteten Rezession werden die einzelnen Veranstaltungen jedoch auch für Juristinnen aus Deutschland von großem Interesse sein. Vorgesehen sind zwei Panels, eines zum Kongressthema und eines zu den Herausforderungen für Gesetzgebung und juristische Praxis in den gegenwärtigen turbulenten Zeiten. Workshops werden sich mit Corporate Social Responsibility, der Finanzkrise und ihren Auswirkungen auf Familien und dem Risikomanagement auf Finanzmärkten befassen. Hinzu kommen Workshops zu Rechtsfragen familiärer Gewalt, zu neuen Geschlechtergleichstellungsstrategien und zum Vertrag von Lissabon.

Abgerundet wird dieses spannende Programm durch ein vielfältiges Rahmenprogramm. Es beginnt bereits am Vorabend der Konferenz mit einem Abendessen in der Blauen Lagune, setzt sich fort mit einer Bootsfahrt zur Insel Videy am ersten Kongressabend und gipfelt in einem halbtätigen Ausflug zum „Goldenen Dreieck“ mit Besuch des Thingvellir, wo sich seit dem 10. Jahrhundert die Volksversammlung traf, und Abstecher zu Geysiren. Eines steht fest: Der Kongress wird ein außergewöhnliches Erlebnis! Das genaue Programm wird Ende Februar auf der Website www.ewla. org veröffentlicht. Eine frühe Anmeldung lohnt sich - wegen der reduzierten „Early Bird“-Teilnahmegebühr und um günstige Flüge zu buchen.

Die Teilnahme steht auch Juristinnen offen, die kein EWLA-Mitglied sind. Allerdings haben EWLA-Mitglieder bei zulassungsbeschränkten Programmpunkten Vorrang ein guter Anlass, EWLA-Mitglied zu werden (bzw. seinen Mitgliedsbeitrag für 2009 zu entrichten)!

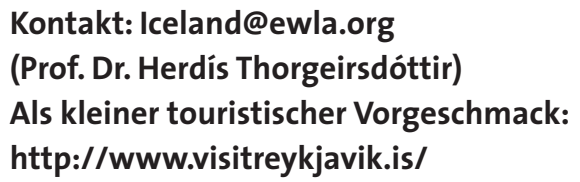

\section{Anwältinnen auf dem DAT 2009}

\section{Anwältinnenkonferenz, 21. und 22. Mai 2009, Stadthalle Braunschweig}

- Prof. Dr. Silke Ruth Laskowski, Universität Kassel, referiert zu,$+=\lesssim$ ? Anspruch und Wirklichkeit - Art. 3 Abs. 2 GG so aktuell wie eh und je“

- Martina Perreng, Referatsleiterin in der Abteilung Arbeits-, Sozial- und Mitbestimmungsrecht beim Bundesvor- stand des DGB, Berlin referiert über „Unmittelbare aber auch versteckte Ungleichbehandlungen von Arbeitnehmerinnen im täglichen Arbeitsleben “

Weitere Informationen:

http://www.dav-anwaeltinnen.de/home.html 